\title{
Complicaciones a corto plazo del cierre percutáneo de comunicación interauricular
}

\author{
Short-term complications of percutaneous atrial septal defect closure \\ Manuel R. Acuña-Fernández*, Adalgisa de Caro, María Maldonado, Milka Marín y Carlos García \\ Instituto Venezolano de los Seguros Sociales, Caracas, Venezuela
}

\section{Resumen}

Introducción: El cierre percutáneo de la comunicación interauricular ostium secundum ha pasado a ser una alternativa a la cirugía convencional. En ese caso, los dispositivos de autocentrado son los más usados entre los cardiólogos intervencionistas. El tipo y la tasa de complicaciones difieren para los distintos dispositivos. Objetivo: Reportar las complicaciones a corto plazo del cierre percutáneo de la comunicación interauricular ostium secundum. Método: Entre abril de 2001 y diciembre de 2017, 129 pacientes (media de edad: 26 años; desviación estándar: 20,39 años) fueron sometidos a cierre percutáneo de comunicación interauricular ostium secundum. Las complicaciones fueron identificadas y clasificadas como mayores y menores. Resultados: 14 pacientes experimentaron complicaciones menores durante la hospitalización y en el primer año de seguimiento (10.85\%), y uno tuvo una complicación mayor que requirió cirugía electiva 4 meses después para la remoción del dispositivo mal posicionado. Entre los que presentaron complicaciones menores, dos tuvieron shunt residual leve, cinco presentaron arritmias interoperatorias, uno refirió dolor torácico y seis tuvieron migraña posimplante. Conclusiones: Esta serie de pacientes muestra que el cierre percutáneo de la comunicación interauricular ostium secundum es un procedimiento seguro y efectivo en casos bien seleccionados. Con el fin de reducir la tasa de complicaciones, están indicados el diagnóstico y el tratamiento tempranos, así como la observación de los criterios de selección de acuerdo con la morfología de la comunicación interauricular ostium secundum. La profilaxis antiagregante con clopidogrel para todos los pacientes requiere mayor investigación.

Palabras clave: Comunicación interauricular. Amplatzer ${ }^{T M}$ Septal Occluder. Detección temprana de enfermedades.

\section{Abstract}

Introduction: Percutaneous closure of ostium secundum atrial septal defects has become an alternative to conventional surgery. Self-centering devices are the ones most used by interventional cardiologists in these cases. The type and rate of complications varies according to the various devices. Objective: To report the short-term complications of percutaneous closure of ostium secundum atrial septal defects. Method: Between April 2001 and December 2017, 129 patients (mean age: 26; standard deviation: 20.39 years) underwent percutaneous closure of ostium secundum atrial septal defects. Complications were identified and classified as major or minor. Two types of devices were used: the Amplatzer ${ }^{T M}$ Septal Occluder in 98 patients, and the Figulla® Septal Occluder in 31 patients. Results:14 patients had minor complications during hospitalization and the first year of follow up (10.85\%), and one had a major complication which required elective surgery four months later

\section{Correspondencia:}

*Manuel R. Acuña-Fernández

E-mail: makuna24@gmail.com
Fecha de recepción: 16-05-2020

Fecha de aceptación: 17-07-2020

DOI: 10.24875/RCCAR.M21000083
Disponible en internet: 29-11-2021 Rev Colomb Cardiol. 2021;28(5):450-457 www.rccardiologia.com 0120-5633 / C 2020 Sociedad Colombiana de Cardiología y Cirugía Cardiovascular. Publicado por Permanyer. Este es un artículo open access bajo la licencia CC BY-NC-ND (http://creativecommons.org/licenses/by-nc-nd/4.0/). 
to remove the misaligned device. Of those who experienced minor complications, two had mild residual shunting, five had intraoperative arrhythmias, one reported chest pain, and six had post-implant migraines. Conclusions: This series of patients shows that percutaneous closure of ostium secundum atrial septal defects is a safe and effective procedure in carefully selected cases. Early diagnosis and treatment are needed to decrease the rate of complications, along with adherence to the selection criteria according to the morphology of the ostium secundum atrial septal defect. Antiplatelet prophylaxis with clopidogrel for all patients requires further study.

Key words: Atrial septal defect. AmplatzerT Septal Occluder. Early disease detection.

\section{Introducción}

Las cardiopatías congénitas son una causa muy frecuente de morbilidad y mortalidad en la edad pediátrica, y no dejan de serlo en la adultez. Dado el carácter variable de la evolución entre individuos, es conveniente planificar el tratamiento definitivo a una edad oportuna $^{1-3}$.

La comunicación interauricular es la segunda cardiopatía congénita más frecuente en la edad pediátrica y la primera en los adultos, y tiene una predominancia marcada por el sexo femenino ${ }^{4-8}$. Cualquier defecto en el tabique interauricular diferente del foramen oval es una comunicación interauricular. Existen tres tipos de comunicación interauricular: a) ostium secundum (60\% de los casos), b) ostium primum y c) seno venoso $0^{9,10}$. De los tres tipos, solo el ostium secundum puede ser ocluido por vía endovascular de manera sistemática. El tratamiento quirúrgico convencional requiere un ingreso hospitalario que oscila entre 3 y 5 días, con un total reintegro del paciente a sus actividades ordinarias a los 30 días de la intervención (deportes y conducción de vehículos). El cierre percutáneo requiere una estancia posoperatoria diez veces menor y con menor morbilidad que el tratamiento quirúrgico. El primer cierre percutáneo de una comunicación interauricular tipo ostium secundum fue realizado por King, Mills y Thompson en abril de 1975, con un dispositivo manufacturado con dos discos de dacrón. Este fue el primer dispositivo implantado en un ser humano y el primero con tecnología de doble disco ${ }^{11}$. Después de 27 años de seguimiento, el caso mencionado fue presentado junto con otros cuatro, que mostraron una excelente evolución. Hace poco más de 20 años, en 1996, en la ciudad de Maracaibo, los doctores Hijazi, Díaz y Hurtado realizaron el primer cierre percutáneo de una comunicación interauricular tipo ostium secundum en Colombia, con un dispositivo Amplatzer ${ }^{\mathrm{TM}}$ Septal Occluder (ASO).

El riesgo quirúrgico y la morbilidad posoperatoria de la cirugía convencional han sido bastante estudiados; en tal sentido, las arritmias son las complicaciones más frecuentes, sobre todo en los adultos ${ }^{7,12}$. La factibilidad y la seguridad del implante de los dispositivos de autocentrado se ha demostrado en adultos y niños, con un porcentaje de oclusión aproximado del 95\%. A pesar de estos resultados, su uso cada vez más asiduo ha develado la aparición de complicaciones potencialmente letales, tales como erosión cardiaca, bloqueo auriculoventricular completo, endocarditis infecciosa y arritmias ${ }^{13-15}$.

Estas complicaciones se han estudiado con mucho detalle, en particular la erosión cardiaca, condición rara que se estima que aparece en menos de 1 en 1000 casos. Por otra parte, el déficit de reborde aórtico $(<5 \mathrm{~mm})$ es la variable que más se relaciona con la erosión tanto precoz como tardía ${ }^{16}$. La endocarditis tras el implante es sumamente rara; sin embargo, de presentarse, requiere la remoción del dispositivo, la reparación del defecto y el tratamiento con antibióticos por 4 a 6 semanas. En un estudio ${ }^{17}$, las complicaciones se estimaron en un $12 \%$, incluyendo las menores y las mayores juntas. Estas se atribuyen a falta de endotelización del dispositivo, pese a que, según las indicaciones del fabricante, la endotelización del ASO ocurre después de 3 meses de su implante ${ }^{18}$.

En cuanto a las arritmias, en su mayoría se describen las intraoperatorias. El bloqueo auriculoventricular completo es infrecuente; hasta 2016 solo se habían descrito cinco casos en el mundo ${ }^{19}$.

Este estudio pretende mostrar las complicaciones de este tipo de procedimiento en los pacientes a quienes se practicó un cierre percutáneo de comunicación interauricular tipo ostium secundum entre el periodo intraoperatorio y el primer año tras el implante.

\section{Método}

Estudio de tipo observacional, retrospectivo y de enfoque positivista, financiado en su totalidad por los investigadores. Se revisó la base de datos del laboratorio de hemodinámica del Hospital Dr. Miguel Pérez 
Carreño, del Instituto Venezolano de los Seguros Sociales, y se seleccionaron pacientes con cierre percutáneo de comunicación interauricular ostium secundum con dispositivo de autocentrado entre 2001 y 2017. Esta investigación cuenta con el aval del comité de ética del hospital en mención. Se analizaron las variables sexo, edad, peso, tipo de dispositivo, diámetro del dispositivo y tiempo de fluoroscopia, y se catalogaron como complicaciones inmediatas y mediatas los siguientes eventos (ocurridos durante el primer año del implante, incluyendo el periodo intraoperatorio, 15 días, 1 mes, 6 meses y 12 meses): a) arritmias durante el procedimiento; b) shunt residual; c) cefalea posterior al implante del dispositivo; y d) implante inadecuado del dispositivo. Las complicaciones en el cierre percutáneo fueron catalogadas como menores y mayores. Las complicaciones menores fueron aquellos eventos transitorios que se resolvieron espontáneamente: arritmia transitoria intraoperatoria, hematoma en la zona de punción, migraña posoperatoria y dolor retroesternal. Se consideraron como complicaciones mayores cualquier condición que comprometiera la vida del paciente, fallecimiento, requerimiento de cirugía periférica o con circulación extracorpórea, y lesión anatómica o funcional permanente como consecuencia del procedimiento $^{13-20}$.

Los dispositivos usados fueron el ASO desde el año 2001 y el Figulla ${ }^{\circledR}$ Septal Occluder (FSO) desde el año 2006, ambos de autocentrado y elaborados con un entramado de nitinol. Estos dispositivos están conformados por dos discos unidos en su porción central por una cintura de conexión. El ASO está elaborado con hilo de nitinol de 0.010 a $0.02032 \mathrm{~mm}$, y cada disco tiene en su interior un parche delgado de poliéster; el disco izquierdo tiene entre 12 y $14 \mathrm{~mm}$ más diámetro que la cintura de conexión y el disco derecho entre 8 y 10 más. Se encuentra disponible en varios tamaños, los cuales están determinados por el diámetro de la cintura de conexión, desde $4 \mathrm{~mm}$ hasta $20 \mathrm{~mm}$ en incrementos de $1 \mathrm{~mm}$, y desde $20 \mathrm{~mm}$ hasta $40 \mathrm{~mm}$ en incrementos de $2 \mathrm{~mm}$. En el centro del disco izquierdo se encuentra un «pin» soldado que une la malla, mientras que en el disco derecho la malla está unida a una microtuerca que se enrosca al microtornillo del cable de entrega.

El FSO es muy similar al ASO. Está constituido por hilo de nitinol de 0.082 a $0.186 \mathrm{~mm}$, y el disco del lado izquierdo es de mayor diámetro que el derecho. La forma del tejido no requiere dos soldaduras, sino solo una ubicada en el centro del disco derecho; en esta descansa una esfera en la cual se fija el cable de entrega (en su más reciente versión, 2007 CE). El dispositivo se encuentra disponible en diferentes tamaños, de 3 a $12 \mathrm{~mm}$, en incrementos de $1,5 \mathrm{~mm}$, y de 15 a $39 \mathrm{~mm}$, en incrementos de $3 \mathrm{~mm}$. Se incluye el diámetro de $40 \mathrm{~mm}^{13-15,21-23}$.

Los criterios de inclusión para el procedimiento fueron diagnóstico definitivo de comunicación interauricular ostium secundum por los siguientes parámetros: a) evidencia clínica, radiológica y ecocardiográfica de sobrecarga de volumen de cavidades derechas; b) presión pulmonar por debajo del $75 \%$ de la presión sistémica; c) bordes del tabique interauricular $>5 \mathrm{~mm}$ (excepto el borde anterior, que podía ser $<5 \mathrm{~mm} 0$ estar ausente); d) medida del stop flow (balón de medida) $\leq 36 \mathrm{~mm} ;$ y e) antecedente de embolia paradójica.

El procedimiento se realiza bajo monitorización ecocardiográfica (transtorácica, transesofágica 0 intracardiaca) y fluoroscópica. Con el paciente anestesiado y anticoagulado con heparina no fraccionada (100 Ul/kg), se accede a través de la vena femoral derecha o izquierda a la aurícula derecha con un catéter multipropósito. Se atraviesa la comunicación interauricular ostium secundum y se accede a la vena pulmonar izquierda superior, preferiblemente, aunque podría ser a la inferior izquierda. A continuación, se introduce una guía extrasoporte de 0.035 pulgadas $/ 260 \mathrm{~cm}$ y sobre esta se desliza un catéter balón de medición que se insufla con contraste diluido en solución estéril, en una proporción 70/30, mediante una jeringa. El tamaño del balón de medición se selecciona de acuerdo con el diámetro del defecto; puede ser de 18, 24 o $34 \mathrm{~mm}$ en el caso del ASO, pero también se usa el balón fabricado por NuMED (Hopkinton, USA), que es muy suave y debe ser inflado poco a poco en el medio del cierre percutáneo de la comunicación interauricular ostium secundum hasta verificar que el flujo mostrado por el Doppler color se detiene (stop flow); de acuerdo con esta medida se selecciona el diámetro del dispositivo. El diámetro del sistema introductor variará según el diámetro del dispositivo seleccionado. Una vez verificada la posición adecuada de la punta del sistema introductor en la aurícula izquierda, se procede a extraer el dispositivo unido al cable de entrega hasta la exposición total del disco izquierdo. Luego, bajo monitorización, fundamentalmente ecocardiográfica, se acerca el disco izquierdo al tabique interauricular. Mediante una suave tracción se fija lo más cerca y paralelo posible al tabique interauricular, y es entonces cuando se libera del disco derecho. Una vez verificada la colocación correcta y la estabilidad del dispositivo, 
se procede a su liberación desenroscando el cable de entrega. En el FSO, el cable de entrega tiene en su extremo distal una pinza que se extrae y captura el pivote ubicado en el centro del disco derecho. Una vez realizado esto, se fija el sistema de seguridad y se procede al cargado del dispositivo, tomando las mismas precauciones que con el ASO. Previa verificación de su estabilidad y fijación, se procede a liberarlo al desplazar las pinzas hacia fuera del cable de entrega. En las figuras 1 a 3 se muestran ambos dispositivos.

Todos los pacientes tuvieron seguimiento clínico, electrocardiográfico y ecocardiográfico transtorácico tras el implante, al día siguiente y a los 15 y 30 días, 6 meses y 12 meses. En todos los casos, excepto en uno, se realizó ecocardiograma transesofágico a los 6 meses. Todos los pacientes egresaron con ácido acetilsalicílico o clopidogrel, o ambos, a dosis de $81 \mathrm{mg} \mathrm{y}$ $75 \mathrm{mg}$, respectivamente; a los pacientes pediátricos se les prescribió ácido acetilsalicílico a dosis de $5 \mathrm{mg} / \mathrm{kg}$ por 6 meses.

Las variables continuas están expresadas con número de pacientes, medianas y medida de los percentiles 25 y 75. Se utilizó la prueba no paramétrica de Mann-Whitney para la comparación de los datos cuantitativos, y la prueba de ji al cuadrado para las variables cualitativas. Se consideró estadísticamente significativo un valor de $p<0.05$. El análisis de la información se realizó mediante el software de Epilnfo (Centers for Disease Control and Prevention, Atlanta, Georgia, USA) y PAST3 (Oslo University, Oslo, Noruega).

\section{Resultados}

En la tabla 1 se muestran las características generales de los pacientes a quienes se realizó el cierre percutáneo con ASO y con FSO. En total hubo 123 casos, 96 con ASO y 27 con FSO. No se siguió un criterio específico de selección de uno u otro dispositivo, salvo la disponibilidad del tamaño adecuado para el paciente. El dispositivo ASO estuvo disponible en Venezuela desde 1998, mientras que el FSO lo estuvo a partir del año 2006. En total se implantaron 96 dispositivos ASO y 27 dispositivos FSO. El periodo de seguimiento fue de 1 año en todos los pacientes. Hubo 14 pacientes con complicaciones menores y un paciente con una complicación mayor.

La mediana de edad fue de 20 años, con un $75 \%$ de los pacientes entre 1 y 46 años. Hubo diferencia significativa entre el grupo de ASO y el de FSO.
Tabla 1. Características de los pacientes ( $n=123$ ), variables estudiadas y seguimiento

\begin{tabular}{|l|c|c|c|}
\hline & ASO (n=96) & FSO (n= 27) & p \\
\hline Edad, M (P 25/75) & $14.5(6.25 / 39)$ & $31(18 / 49)$ & 0.009 \\
\hline Sexo (\%) & $\begin{array}{l}\mathrm{F}=67(68.7 \%) \\
\mathrm{M}=30(31.3 \%)\end{array}$ & $\begin{array}{l}\mathrm{F}=21(77.9 \%) \\
\mathrm{M}=6(22.1 \%)\end{array}$ & 0.83 \\
\hline $\begin{array}{l}\text { Peso, } \mathrm{kg}, \mathrm{M} \text { (P } \\
25 / 75)\end{array}$ & $40(18 / 57.5)$ & $57(49 / 63)$ & 0.003 \\
\hline $\begin{array}{l}\text { Dispositivo, mm } \varnothing \\
\text { (P 25/75) }\end{array}$ & $22(15 / 32)$ & $24(18 / 30)$ & 0.70 \\
\hline \begin{tabular}{l} 
Shunt residual, $\mathrm{n}$ \\
\hline Arritmias, $\mathrm{n}$
\end{tabular} & 1 & 2 & $\mathrm{NS}$ \\
\hline $\begin{array}{l}\text { Dolor toracico, } \mathrm{n} \\
\text { Cefalea } \\
\text { posimplante, } \mathrm{n}\end{array}$ & 1 & 0 & \\
\hline $\begin{array}{l}\text { Tiempo de } \\
\text { fluoroscopia, min }\end{array}$ & $10(6.13 / 17.18)$ & $6.5(3.9 / 8.4)$ & 0.001 \\
\hline
\end{tabular}

ASO: AmplatzerTM Septal Occluder; FSO: Figulla® Septal 0ccluder; NS: no significativo.

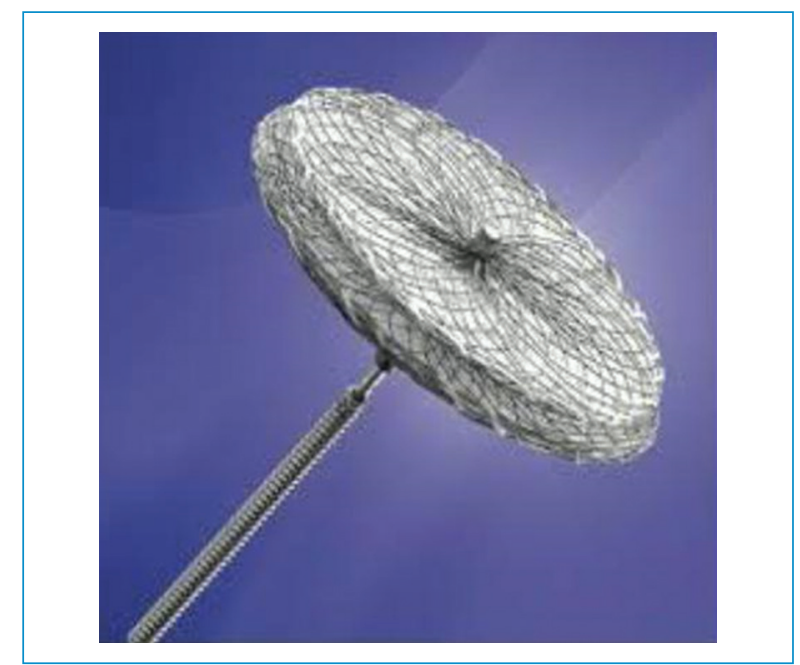

Figura 1. Dispositivo Amplatzer ${ }^{\mathrm{TM}}$ (Abbot Laboratories, Chicago, Illinois, USA).

En cuanto a la proporción de casos según el sexo, $90(70.3 \%)$ correspondieron al femenino y $38(29.7 \%)$ al masculino, con una relación 2.37:1.

La mediana de peso fue de $42.4 \mathrm{~kg}$, con el $75 \%$ los pacientes entre 9.5 y $61 \mathrm{~kg}$ ( $(25 / 75:$ 19/61 kg).

La mediana del diámetro del dispositivo seleccionado fue de $22 \mathrm{~mm}$, en el $75 \%$ de los casos entre 6 y $30 \mathrm{~mm}$ (P 25/75: 15/30 mm). Se implantaron dos 


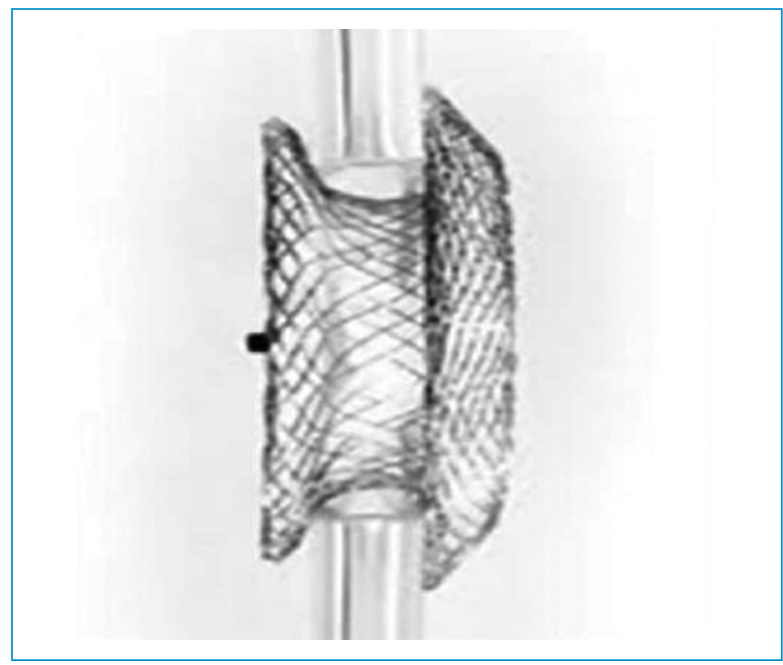

Figura 2. Dispositivo Amplatzer ${ }^{\mathrm{TM}}$ (Abbot Laboratories, Chicago, Illinois, USA).

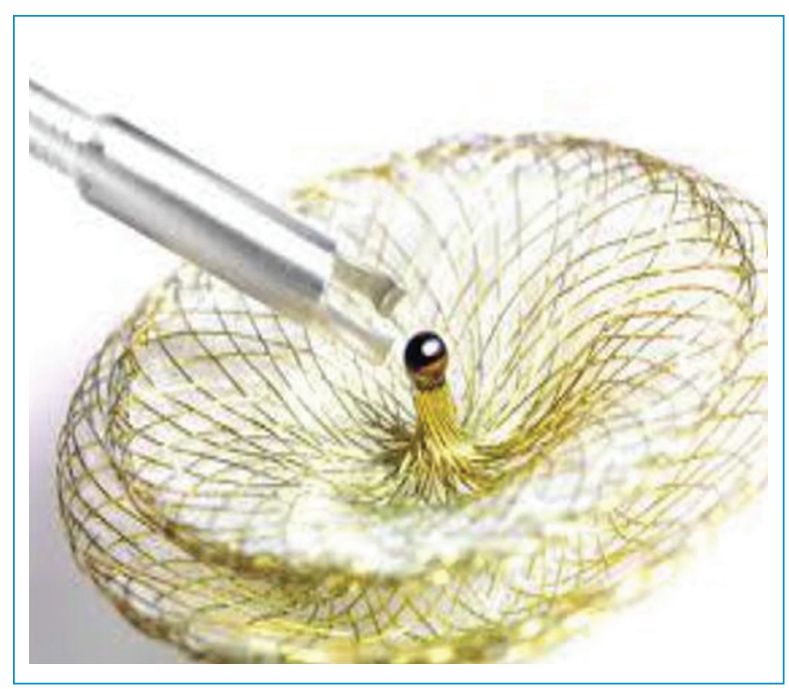

Figura 3. Figulla ${ }^{\circledR}$ Flex (Occlutech AB, Helsinborg, Suecia).

dispositivos de diámetro $26 \mathrm{~mm}$ y cuatro de diámetro $40 \mathrm{~mm}$.

El comportamiento de la variable shunt residual fue un dato interesante para los objetivos de la investigación. Este pudo ser evaluado al realizar el ecocardiograma sistemático después de liberar el dispositivo mediante la técnica de Doppler color. Adicionalmente, a todos los pacientes se les realizaron controles ecocardiográficos a los 15 y 30 días, y a los $1,3,6$ y 12 meses. Solo un paciente mostró shunt residual con un dispositivo ASO de diámetro $21 \mathrm{~mm}$ en el primer año de seguimiento, y en el grupo de FSO hubo dos casos de shunt residual.
Se documentaron arritmias transitorias y de morfología supraventricular en 5 (4,06\%) pacientes. Un paciente requirió la administración de una dosis de $2 \mathrm{mg}$ de verapamilo por vía intravenosa.

Un solo paciente presentó dolor torácico retroesternal por primera vez y sin irradiación en el posoperatorio inmediato de implante del ASO.

La cefalea tras el implante es un síntoma frecuente en este tipo de procedimiento. En esta investigación se presentaron seis casos $(4.65 \%)$, en cuatro mujeres (3.1\%) y dos hombres (1.55\%); de ellos, solo uno era de edad pediátrica. Todos los pacientes respondieron a analgésicos comunes y en el $100 \%$ la cefalea desapareció al sexto mes de control. Se halló una diferencia estadísticamente significativa entre las mujeres y los hombres $(p=0.009)$, siendo más frecuente en ellas.

El tiempo total de fluoroscopia da idea de la complejidad de un procedimiento de cardiología intervencionista, pues incluye la fluoroscopia, la visualización y la grabación o cine, que son reflejo de la manipulación de los catéteres, las guías y el dispositivo desde el comienzo hasta el final. En la muestra general, la mediana del tiempo de fluoroscopia fue de $11.7 \mathrm{~min}$ (P 25/75: 5.73/15.3). Se halló una diferencia significativa $(p<0.001)$ en la relación entre el peso de los pacientes y el tiempo total de fluoroscopia, siendo menor el tiempo total de fluoroscopia en los pacientes con mayor peso. Se estableció una diferencia significativa en el tiempo total de fluoroscopia del grupo ASO, con una mediana de 13.6 minutos (2.7-49.9), frente al grupo FSO, con una mediana de 6.25 minutos (3.2-16.3; $\mathrm{p}=0.0026)$. Sin embargo, al comparar los últimos 27 casos de la serie de ASO (P 25/75: 4.06/8.6) con los de FSO (P 25/75: 3.9/8.4), la diferencia no fue significativa $(p=0.70)$. Se realizó esta comparación entre implantes para establecer una mayor objetividad en los datos.

\section{Discusión}

Las complicaciones posoperatorias del cierre percutáneo de la comunicación interauricular ostium secundum se han clasificado cronológicamente en tempranas, intermedias y tardías, o simplemente a corto, mediano y largo plazo, y de acuerdo con su complejidad, en mayores y menores ${ }^{13}$.

En este estudio se muestra la frecuencia de las complicaciones a corto plazo (1 año) del cierre percutáneo de la comunicación interauricular ostium secundum mediante un dispositivo de doble disco, sea ASO o FSO. Ambos dispositivos pueden ser entregados y 
recapturados hasta su colocación adecuada para ser finalmente liberados. La técnica de implante y su forma son muy similares, salvo por el tipo de cable de entrega y su mecanismo de liberación, por lo que no se considera necesaria una curva de aprendizaje para el uso del FSO dada su similitud con el $\mathrm{ASO}^{22,23}$. La mayor cantidad de casos de ASO que de FSO, así como la diferencia significativa respecto a la edad en esta investigación, no se deben al azar, sino a la disponibilidad de los últimos en el mercado local a partir de 2009.

La edad mediana de los pacientes de esta serie es de 20 años, lo cual ratifica el hecho de que esta condición se manifiesta de manera tardía, relativamente con pocos síntomas y en su mayoría detectados en la edad adulta por una disminución en la capacidad aérobica; en la infancia solo se detecta por escasa ganancia ponderal. Tal como ya ha sido reportado, esta afección es mucho más frecuente en el sexo femeni$\mathrm{no}^{4-8,10}$. Así mismo, tiene un fondo genético por alteración del gen NKX2-524,25. En la población estudiada, la distribución de los casos según el sexo fue similar a lo descrito en otros estudios: 2:126. Dado que el diagnóstico y el tratamiento percutáneo se realizan usualmente sin la administración de contraste yodado, el hipotiroidismo secundario en niños, como complicación posoperatoria, es nulo ${ }^{27}$.

En cuanto al peso, la media y la mediana ubican a los pacientes en 45 y $42 \mathrm{~kg}$. La repercusión hemodinámica que influiría en la ganancia ponderal está relacionada, a su vez, con la distensibilidad del ventrículo derecho (VD). Cuanta menos edad tenían los pacientes pediátricos, más repercusión tenía en el peso, lo cual constituyó el motivo de su intervención antes de los 5 años. Sin embargo, la mayor distensibilidad del VD en algunos casos es lo que producirá la ausencia de síntomas hasta la tercera o cuarta décadas de la vida, lo cual explicaría casos de desarrollo pondoestatural adecuado. Después del cierre percutáneo, la reducción del tamaño de las cavidades derechas se ha descrito como significativa, aunque varía de un paciente a otro $^{28,29}$.

En cuanto al diámetro del dispositivo, el $75 \%$ de los casos requirieron uno de $30 \mathrm{~mm}$ o menos, lo cual es relevante para el mantenimiento de un stock en el laboratorio de hemodinámica con un criterio de gestión apropiado. El dispositivo más usado fue el de $24 \mathrm{~mm}$, lo cual coincide con lo descrito en otro estudio ${ }^{17}$. Aunque no se reportó ningún caso de erosión durante el seguimiento, se han descrito de forma tardía ${ }^{15,29}$. Desde el punto de vista estadístico, el diámetro del dispositivo más relacionado con erosión cardíaca es el de $26 \mathrm{~mm}^{15}$.
En general, la presencia de shunt residual se ha descrito como baja, del $9 \%^{30,31}$; sin embargo, después de 6 meses se observó en el $2.32 \%$ de los pacientes de nuestro estudio. La selección correcta de los casos y evitar el sobredimensionamiento del dispositivo podrían minimizar la presencia de shunt residual. Por tanto, es conveniente realizar, después de 6 meses del implante, un ecocardiograma transesofágico o transtorácico con contraste para identificar a estos pacientes, ya que un orificio restrictivo adyacente al defecto principal puede pasar inadvertido al momento del implante. Uno de los casos requirió cirugía debido a dislocación del disco izquierdo, por lo que se optó por retirarlo mediante cirugía y se hizo septoplastia con parche de pericardio bovino. En el grupo de FSO, dos pacientes presentaron shunt residual; en uno se debió a hipertensión pulmonar moderada y por ello se realizó fenestración en el dispositivo para descompresión (la fenestración cerró espontáneamente en el primer año de evolución), y el otro fue un shunt residual leve, que permaneció durante el primer año.

Aunque no se registró una reducción en el diámetro del VD como variable, esta es más acelerada en los pacientes más jóvenes ${ }^{29}$. La dilatación de cavidades cardiacas se relaciona con la aparición de arritmias, de modo que la desaparición del shunt ocasiona regresión del diámetro del VD y podría prevenir el desarrollo futuro de fibrilación o flutter. En la serie estudiada, una paciente afectada por flutter retornó a ritmo sinusal durante el seguimiento, lo cual concuerda con lo descrito en la literatura $^{29,32}$. La mayoría de las arritmias encontradas durante el posoperatorio fueron de morfología supraventricular y autolimitadas ${ }^{33,34}$. La descripción de arritmias supraventriculares varía de un estudio a otro, y van desde el $0 \%$ hasta el $4.7 \%$. En nuestro estudio se presentaron en el $4.06 \%$ y solo un caso requirió, en el intraoperatorio, la administración de verapamilo, con lo que se obtuvo una respuesta adecuada.

Cuando se evaluó la presencia de dolor torácico se encontró un solo paciente: una mujer de 47 años y $87 \mathrm{~kg}$, con un tiempo de fluoroscopia de 4 minutos (procedimiento expedito, sin complicaciones). De inmediato se realizaron electrocardiograma y ecocardiograma transtorácico y transesofágico, en los que no se observó ninguna anormalidad. A la semana siguiente del implante se le realizaron coronariografía y prueba de esfuerzo, las cuales fueron negativas para erosión, anomalías coronarias e isquemia inducida por el ejercicio, respectivamente. El dolor desapareció de manera espontánea y la paciente tuvo una evolución asintomática durante el resto del seguimiento. Este 
síntoma merece especial atención, ya que puede ser indicio de erosión o de compromiso en el flujo coronario. En este caso no se pudo identificar su origen del mismo. Algunos pacientes mencionan, de forma anecdótica y muy pasajera, cierto disconfort retroesternal, el cual ha sido relacionado con el trauma producido por la sonda transesofágica ${ }^{34}$.

Por otro lado, se evidenció una mayor frecuencia de cefalea tras el implante en el sexo femenino. Se ha propuesto que su génesis radica en la activación plaquetaria y la liberación de histamina debido a la presencia del dispositivo, un cuerpo extraño, en el corazón. La frecuencia de cefalea posimplante en la edad pediátrica fue muy baja en la serie estudiada, del $0.78 \%$; sin embargo, en el grupo general fue del $4.65 \%$. Algunos autores han relacionado el tamaño del dispositivo con la aparición de migraña de novo sin aura, pero otros no han encontrado coincidencia ${ }^{35-37}$. Se ha propuesto que el mecanismo de la aparición de la migraña o cefalea sea la activación plaquetaria y la subsecuente liberación de grandes cantidades de serotonina.

En ningún paciente se detectó trombo sobre la superficie del dispositivo; sin embargo, en algunos casos se han evidenciado microémbolos, los cuales provocan depresión cortical difusa ${ }^{38,39}$. La deformación o el estrechamiento que puede producir el dispositivo puede inducir la liberación de sustancias vasoactivas que están asociadas con la aparición de migraña con aura, tales como el péptido relacionado con el gen de la calcitonina y el péptido atrial natriurético ${ }^{35-39}$.

En general, el tiempo de fluoroscopia es corto y, como en cualquier procedimiento, la curva de aprendizaje del equipo de trabajo contribuye a la minimización de riesgos. El promedio del tiempo de radiación es de 11.63 minutos; pese a ello, se apreció una exposición $\tan$ baja como 1.6 minutos. El método puede ser igual de eficiente en cualquier edad, incluso en pacientes de 70 años, lo cual se refleja en unos bajos tiempos de exposición a radiación ${ }^{40,41}$. Es de notar que, en la medida en que el equipo de trabajo del laboratorio de hemodinámica está más familiarizado con el procedimiento, más disminuye el tiempo de fluoroscopia, al punto de poder hacerse sin esta ${ }^{41-43}$.

Se ha publicado la práctica sistemática de una técnica híbrida: mediante una toracotomía derecha submamaria de $3 \mathrm{~cm}$ se expone el pericardio y, luego de suspenderlo, se realiza una jareta de $1.5-2 \mathrm{~cm}$ en la aurícula derecha. A través de esta, se introduce la vaina de entrega y se implanta el dispositivo. Todo el procedimiento se lleva a cabo con ecocardiografía transtorácica 0 transesofágica ${ }^{43}$. Para los autores del presente artículo, la técnica híbrida solo debe considerarse en casos de ausencia de vena cava inferior.

El tiempo de fluoroscopia fue menor en los pacientes de mayor peso, lo cual se atribuye a la mayor facilidad para manipular el dispositivo en un corazón de gran tamaño. Los tiempos de fluoroscopia fueron menores con el FSO que con el ASO, tal vez por la similitud en el diseño de ambos dispositivos y por la casi idéntica técnica de implante; de ahí que la curva de aprendizaje del FSO fuera cubierta por el $\mathrm{ASO}^{23}$.

La limitante del estudio fue la no aleatorización de la asignación del dispositivo a utilizar en cada caso.

\section{Conclusiones}

Con esta experiencia es posible determinar que el cierre percutáneo de la comunicación interauricular tipo ostium secundum es un método eficiente tanto en niños como en adultos. Adicionalmente, las complicaciones a corto plazo son escasas, de fácil manejo y corrección, y las arritmias son, en su mayoría, de aparición intraoperatoria y de curso benigno.

Cabe destacar la importancia de un diagnóstico precoz, con el objetivo de tratar oportunamente la enfermedad y evitar complicaciones producto de la sobrecarga de volumen cardiaco, ya que la comunicación interauricular ostium secundum es oligosintomática en la infancia. Así mismo, es pertinente enfatizar, tanto en pediatras como en internistas, que la mejor estrategia terapéutica es la endovascular sobre la quirúrgica, por lo que estos pacientes deben ser derivados a cardiología intervencionista y no a cirugía cardiovascular.

Por otro lado, se debe insistir a los pacientes en mantener el control médico por el centro de implante, ya que existen complicaciones a largo plazo que deben ser monitorizadas, al igual que ocurre en una cirugía convencional.

En los laboratorios de hemodinámica es recomendable disponer de más de un tipo de tecnología para el tratamiento de los pacientes con cierre percutáneo de la comunicación interauricular ostium secundum, pues lo que para unos puede ser una excelente alternativa, para otros no lo será.

Por último, se recomienda que los médicos hemodinamistas conozcan bien los posibles riesgos de la exposición al tratamiento de los pacientes, con miras a prevenir desenlaces fatales, incluso en casos sencillos.

\section{Financiamiento}

Financiamiento a cargo de los investigadores. 


\section{Conflicto de intereses}

Los autores declaran no tener conflicto de intereses.

\section{Responsabilidades éticas}

Protección de personas y animales. Los autores declaran que para esta investigación no se han realizado experimentos en seres humanos ni en animales.

Confidencialidad de los datos. Los autores declaran que han seguido los protocolos de su centro de trabajo sobre la publicación de datos de pacientes.

Derecho a la privacidad y consentimiento informado. Los autores declaran que en este artículo no aparecen datos de pacientes.

\section{Bibliografía}

1. Bravo-Valenzuela NJ, Peixoto AB, Araujo Júnior E. Prenatal diagnosis of congenital heart disease. A review of current knowledge. Indian Jeart J. 2018;70:150-64.

2. Sharma S, Kaur N, Kaur K, Pawar NC. Role of echocardiography in prenatal screening of congenital heart disease and its correlation with prenatal outcome. J Clin Diagn Res. 2017;11:12-4.

3. Ferencz C, Rubin JD, McCarter RJ, Brenner JI, Neill CA, Perry LW, et al. Prevalence at livebirth at the Baltimore-Washington Infant Study. Am J Epidemiol. 1985;121:31-6.

4. Carlgren LE. The incidence of congenital heart disease in children born in Gottenburg, 1941-1950. Br Heart J. 1959;21:40-50.

5. Hoffman J, Kaplan S. The incidence of congenital heart disease. Am Coll Cardiol. 2002;39:1890-900.

6. Espino Vela J, Toro A. Natural history of atrial septal defect. Cardiovasc Clin. 1971;2:104-25.

7. Rahitoola SF, Kirkling JW, Burchell HB. Atrial septal defect. Circulation. 1968;67(Suppl 5):2-12.

8. van Velzen CL, Clur SA, Rijlaarsdam ME, Bax CJ, Pajkrt E, Heymans MW, et al. Prenatal detection of congenital heart disease - results of a national screening programme. BJOG. 2016;123:400-7.

9. Wesley Vick G, Titus J. Defects of the atrial septum including the atrioventricular canal in the science and practice of pediatric cardiology. En Garson A, Bricker T, Mcnamara D, editores. Philadelphia/London: Lea \& Febiger; 1990. p. 1203-35.

10. Roesler H. Interatrial septal defect. Arch Intern Med. 1934;54:339-80.

11. King TD, Mills N. Historical perspectives. En: Hijazi Z, Feldman T, Mustafa, Al-Qbandi A, Sievert H, editors. Transcatheter closure of ASDs and PFOs. A comprehensive assessment. Minneapolis, USA: Cardiotext Publishing; 2010. p. 41-2.

12. Dickinson DF, Arnold R, Wilkinson JL. Congenital heart disease among 16048 live born children in Liverpool, 1960 to 1969. Implications for surgical treatment. Br Heart J. 1981;16:55-2.

13. Chessa M, Carminati M, Butera G, Bini BM, Drago M, Rosti L, et al. Early and late complications associated with transcatheter occlusion of secundum atrial defect. J Am Coll Cardiol. 2002;39:1061-5.

14. Harper R, Mottram PM, McGraw DJ. Closure of secundum atrial defects with the Amplatzer septal occluder device: techniques and problems. Catheter Cardiovasc Interv. 2002;57:508-24.

15. Amin Z, Hijazi ZM, Bass JL, Cheatham JP, Hellenbrand WE, Kleinman CS Erosion of Amplatzer septal occluder device after closure of secundum atrial defect. Review of registry of complications and recommendations to minimize future disc erosions. Catheter Cardiovasc Interv. 2004;63:496-502.

16. McElhinney D, Michael D, Kenny D, Alboliras E, Amin Z. Relative risk factors for cardiac erosion following transcatheter closure of atrial septal defects. Circulation. 2016;113:1738-46.

17. Snijder R, Renes L, Bosshardt D, Suttorp M, Berg J, Post M. Percutaneous atrial septal defect closure using the Occlutech Figulla device in adults: more than 800 patient-years of follow-up. J Interv Cardiol. 2020;2020:7136802.
18. Kim DJ, Shim CY, You SC, Lee SH, Hong GR. Late bacterial endocarditis and abscess formation after implantation of an Amplatzer septal occluder device. Circulation. 2015;131:e536-8.

19. Jalal Z, Hascoet S, Baruteau A, Iriart X, Kreitmann B, Boudjemline $Y$, et al. Long-term complications after transcatheter atrial septal defect closure: a review of the medical literature. Can J Cardiol. 2016;32:1315. e11-8.

20. Jalal Z, Hascoët S, Gronier C, Godart F, Mauri L, Dauphin C. Long-term outcomes after percutaneous closure of ostium secundum atrial septal defect in the young. JACC Cardiovasc Interv. 2018;11:795-804.

21. Rao PS, Harris AD. Recent advances in managing septal defects: atrial septal defects. F1000Res. 2017;6:20-42.

22. Pac A, Polat TB, Cetin I, Oflaz MB, Balli S. Figulla ASD occluder versus Amplatzer septal occluder: a comparative study on validation of a novel device for percutaneous closure of atrial septal defects. J Interv Cardiol. 2009;22:489-95.

23. Godart F, Houeijeh A, Recher M, Francart C, Polge AS, Richardson M, et al. Transcatheter closure of atrial septal defect with the Figulla® ASD Occluder: a comparative study with the Amplatzer® Septal Occluder. Arch Cardiovasc Dis. 2015;108:57-63.

24. Chung I, Rajakumar G. Genetics of congenital heart defects: the NKX25 gene, a key player. Genes. 2016;7:6.

25. Dayal D, Prasad R. Congenital hypothyroidism: current perspectives. Res Rep Endocr Disord. 2015;5:91-102.

26. Wilmshurst PT, Pearson MJ, Nightingale S, Walsh KP, Morrison WL. Inheritance of persistent foramen ovale and atrial septal defects and the relation to familial migraine with aura. Heart. 2004;90:1315-20.

27. Thaker VV, Galler MF, Marshall AC, Almodovar MC, Hsu H-W, Addis CJ, et al. Hypothyroidism in infants with congenital heart disease exposed to excess iodine. J Endocr Soc. 2017;1:1067-78.

28. Mostafa S, Abdelhakim A, Aboelazm T, Arafa O, Elemam A. Effect of transcatheter closure of secundum atrial septal defect on cardiac electric remodeling. Int J Hear Rhythm. 2017;2:40-8.

29. Bishnoi RN, Everett AD, Ringel RE, Owada CY, Rhodes JF, John J, et al. Device closure of secundum atrial septal defects in infants weighing less than $8 \mathrm{~kg}$. Pediatr Cardiol. 2014;35:1124-31.

30. Zuñiga Luna M, Gallego Muñoz C, Vásquez Villegas S, Rendón Isaza JC, Uribe CE. Erosión muy tardía de la aurícula derecha con dispositivo Amplatzer®; reporte de un caso. Arch Cardiol Mex. 2016;86:271-5.

31. Krasuski RA, Zdradzinski M, Lee J, Elkin R, El Mallah W. Impact of device type on residual shunt following percutaneous atrial septal defect closure. J Am Coll Cardiol. 2016:67:933.

32. Ströker E, Van De Bruaene A, De Meester P, Van Deyck K, Gewllig M, Budts W. Transcatheter device closure of atrial septal defects in patients above age 60. Acta Cardiol. 2013;68:127-32.

33. Brandenburg RO, Holmes Jr DR, Brandenburg RO, McGoon DC. Clinical follow up study of paroxysmal supraventricular tachyarrhythmias after operative repair of a secundum type atrial septal defects in adults. Am J Card. 1983;51:273-6.

34. Mathur SK, Pooja S. Transesophageal echocardiography related complications. Indian J Anaesth. 2009;53:567-74.

35. Kato $Y$, Furuya D, Ishido $H$, Kobayashi T, Tanahashi N. New-onset migraine with aura after transcatheter closure of atrial septal defect. J Headache Pain. 2012;13:493-5.

36. Kato $\mathrm{Y}$, Kobayashi T, Ishido H, Hayashi T, Furuya D, Tanahashi N. Migraine attacks after transcatheter closure of atrial septal defect. Cephalalgia. 2013;33:1229-37.

37. Mortelmans K, Post M, Thijs V, Herroelen L, Budts W. The influence of percutaneous atrial septal defect closure on the occurrence of migraine. Eur Heart J. 2005;26:1533-7.

38. Ferrari J, Baumgartner $\mathrm{H}$, Tentschert S, Dorda V, Lang W, Willfort-Ehringer $\mathrm{A}$, et al. Cerebral microembolism during transcatheter closure of patent foramen ovale. J Neurol. 2004:251:825-9.

39. Wei SH, Fan PC, Kuo PH, Chiou LC, Wang JK. Calcitonin gene-related peptide and size of the atrial septal defect in new-onset migraine after transcatheter closure: results of a preliminary study. Headache. 2012;52:985-92

40. Khan AA, Tan JL, Li W, Dimopoulos K, Spence MS, Chow P, et al. The impact of transcatheter atrial septal defect closure in the older population. A prospective study. JACC Cardiovasc Interv. 2010;3:276-81.

41. Yang Y, Zhang W, Wu Q, Gao L, Jin W, Zhao T. Transcatheter closure of atrial septal defects without fluoroscopy: a well-established procedure for alternative use in children. Eurolntervention. 2016;12:e652-7.

42. Zanjani KS, Zeinaloo A, Malekan-Rad E. Transcatheter atrial septal defect closure under transthorasic echocardiography in children. Iran J Pediatr. 2011;21:473-8.

43. Han-Fan Q, Qiang C, Zhi-Nuan H, Liang-Wan C, Xue-Shan H. Transcatheter and intraoperative device closure and surgical repair for atrial septal defect. J Cardiothorac Surg. 2019;14:136. 